\title{
A "Building Block" Approach to Application Development for Education and Decision Support in Radiology: Implications for Integrated Clinical Information Systems Environments
}

\author{
Robert A. Greenes
}

\begin{abstract}
Education and decision-support resources useful to radiologists are proliferating for the personal computer/workstation user or are potentially accessible via high-speed networks. These resources are typically made available through a set of application programs that tend to be developed in isolation and operate independently. Nonetheless, there is a growing need for an integrated environment for access to these resources in the context of professional work, during clinical problem-solving and decision-making activities, and for use in conjunction with other information resources. New application development environments are required to provide these capabilities. One such architecture for applications, which we have implemented in a prototype environment called DeSyGNER, is based on separately delineating the component information resources required for an application, termed entities, and the user interface and organizational paradigms, or composition methods, by which the entities are used to provide particular kinds of capability. Examples include composition methods to support query, book browsing, hyperlinking, tutorials, simulations, or question/answer testing. Future steps must address true integration of such applications with existing clinical information systems. We believe that the most viable approach for evolving this capability is based on the use of new software engineering methodologies, open systems, client-server communication, and delineation of standard message protocols.
\end{abstract}

Copyright $\odot 1991$ by W.B. Saunders Company

KEY WORDS: information system architecture, computer-aided decision support, computer-based education, authoring tools, radiological information needs.

$\mathbf{O}$ CCASSIONS frequently arise in the practice of radiology in which it would be useful to access information resources that provide educational and decision-making assistance. While these resources may be helpful on a stand-alone basis, the need for them often occurs in the context of doing other professional work (eg, interpreting a patient's examination or consulting with a referring physician about the next steps in workup and management).

Potentially valuable information resources are proliferating owing to the flexibility and power of personal computers and workstations, on which considerable application development has been focused, the appearance of user- friendly authoring languages for educational and multimedia applications, and high speed networking. The latter is making it increasingly feasible to access applications not available on one's local computer.

Useful resources encompass miriad capabilities, ${ }^{1}$ including bibliographic databases, electronic textbook materials, three-dimensional models and atlases, heuristic or probabilistic inference tools, libraries of archived images with known diagnoses, question/answer databanks, and clinical practice guidelines or protocols for specific problems. They represent a diverse collection of media, formats, and methodologies, including both passive information resources (text, tables, charts, images, motion sequences, etc) and adaptive resources (simulations, analytic tools, expert systems, etc).

Such resources may be used in a variety of application contexts (eg, targeted problem solving, pursuit of curiosity, browsing, directed learning, drill and practice, and testing/self-assessment). A single application may use multiple types of resources. Different applications may, furthermore, have need for the same resources but use them differently, "repurposing" them.

From a user's point of view, however, needed resources are often available only through isolated programs, possibly on disparate and even incompatible computers. Applications aimed at similar function may have different user interfaces and methods of interaction. There is limited ability to carry out complex problem solving activities by combining functions of different applications or by passing data from one to the next.

From the Decision Systems Group, Hanard Medical School, Brigham and Women's Hospital, Boston, MA.

Supported in part by Grants LM 04572 and LM 07037 from the National Library of Medicine, and Grant CA 45574 from the National Cancer Institute.

Address reprint requests to Dr Robert A. Greenes, Department of Radiology, Brigham and Women's Hospital, 75

Francis St, Boston, MA 02115 .

Copyright $₫ 1991$ by W.B. Saunders Company

0897-1889/91/0404-0001\$03.00/0 
From the authoring point of view, useful content (eg, a diagram, an image, or text) is often embedded in applications, accessible only via the application itself, or in applicationspecific data formats. Consequently, much duplication of effort occurs, and applications with wide-ranging or comprehensive goals often cannot build on content or capabilities contained in other relevant applications. This limits the scope and usefulness of applications development. Developers are destined to continually "reinvent the wheel."

For these reasons, we have been interested in application development based on a building block approach. We believe it is desirable to separate the construction of applications into two aspects: (1) the individual information resources used and (2) the organizational framework for viewing and interacting with them. We have designed and implemented a new software development architecture for construction of workstàtion-oriented applications from modular, distributed network resources. This software architecture, known as DeSyGNER (Decision Systems Group Nucleus of Extensible Resources), ${ }^{2,3}$ has been applied primarily to education and decision-support applications.

In this article we characterize the various needs of radiologists for education and decision support, in terms of information resources required, and describe how DeSyGNER is applied to meeting these needs. We then address what we consider to be a major issue that impedes use of educational and decision support applications in practice, that is, the difficulties of integrating them with clinical applications. We conclude with a discussion of some of the principles that must be addressed for such integration to occur, which form a basis for our continuing and future work.

\section{EDUCATION AND DECISION-SUPPORT NEEDS IN RADIOLOGIC PRACTICE}

Reflection on the professional work environment of the radiologist can help to determine the optimal way in which education and decisionsupport resources should be integrated into practice, and the requirements for doing so. We consider several scenarios illustrating aspects of an idealized work environment.

\section{Illustrative Scenarios}

Consider the radiologist in the process of interpreting an examination. Depending on the clinical findings and indication for the examination, the radiologist may wish to access other information about the patient, such as prior imaging studies, laboratory tests, and problem list. He or she may find it useful to consult a decision aid to provide a differential diagnosis of a particular finding that is visualized, review similar images in an archived teaching file, or request a consultation from a colleague before rendering an interpretation. The radiologist may wish to save the case for follow-up, teaching, or research. Ideally, subsequent feedback to the radiologist should occur automatically and include notification of the results of biopsies, surgery, or more definitive studies of the area in question.

As another example, the referring physician reviewing a radiologic report may wish to view the images directly and go over the findings and interpretation with the radiologist, and perhaps request advice from the radiologist about further workup. This might include review and discussion of available guidelines for this problem, explanations, exceptions, and pertinent references. In complex circumstances, it may even be desirable to perform a decision analysis exploring the tradeoffs associated with alternative strategies.

Figure 1 shows the manner in which existing information resources and applications could be used, in conjunction with one another, to provide support for several aspects of both of the above scenarios.

In educational settings, including both training and continuing education, a radiologist may wish to study interactive tutorials or take selfassessment quizzes, in which cases are viewed, diagnoses are attempted, and feedback is provided. As in the actual clinical setting, the feedback should include access to differential diagnosis information, related images, disease descriptions, pathophysiologic discussions, and pertinent references. The radiologist may wish to browse anatomic atlases, review threedimensional models, or electronic textbooks and journals pertaining to a specific topic of interest.

For clinical research, a radiologist should 


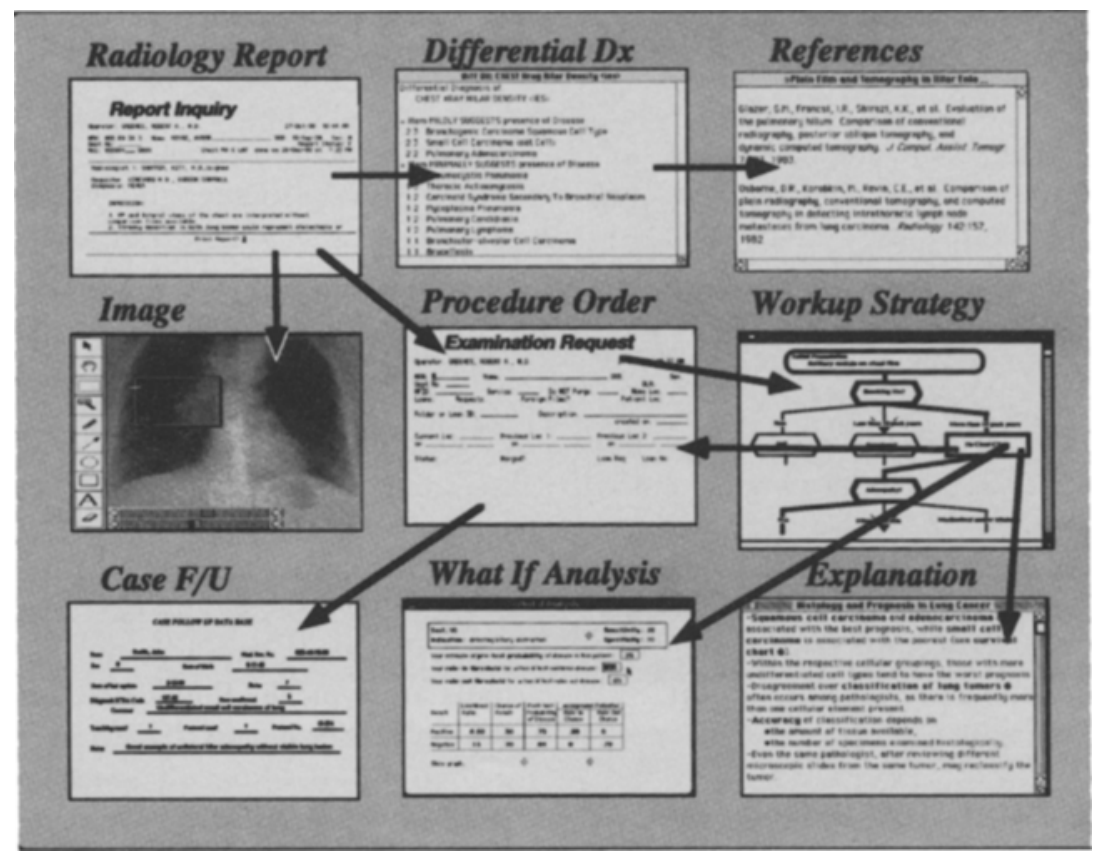

Fig 1. Potentially useful information resources to which a radiologist may desire access while interpreting an examination, or that a referring clinician might find useful while reviewing a report. In the context of composing (or reviewing) a radiology report, the user may wish to obtain a differential diagnosis for a finding, or examine the image for the case (or typical images associated with diseases in the differential). References may be desired. In considering the next steps in patient management, a diagnostic algorithm may be consulted, explanatory notes reviewed, and a "what if" analysis performed to determine potential benefit of a contemplated test. Interesting or puzzling cases may be marked for subsequent follow-up and perhaps automatic feedback of the results of further testing. Note that screen displays in this figure normally include color, for specific content or for highlighting, although reproduced here in black and white.

have the means during routine practice to designate and segregate for subsequent study certain cases of particular interest. For research, the radiologist should then be able to access the associated images, as well as related clinical information, record these items in a personal database, perform various manipulations, including tabulations and statistical analyses, and display the results in tables and graphs. These may then be organized into manuscripts and slide presentations through the use of other tools. Or they may become incorporated in additional educational and decision-support applications.

\section{Information Tasks of the Radiologist}

Elsewhere we have analyzed the radiologic process in terms of eight distinct component activities $^{4}$ and their information requirements. These activities are all information intensive, and involve access to several distinct computing environments. However, the activities are per- formed by a variety of individuals, the radiologist among them, rather than a single individual.

Here, we wish to take a different perspective, focusing on the radiologist. Health-care professionals such as the radiologist have information needs that are different from those of nonprofessionals in terms of the diversity of tasks performed and the importance of integration of information from diverse sources. ${ }^{5}$ To some extent, professional activities of the radiologist can be classified as being either clinical practiceoriented (ie, involved in the radiologic process) or non-practice-oriented. The primary information tasks associated with these two classes of activity are listed as follows.

Clinical practice-oriented activities. Clinical practice-oriented activities include * patient data access and determination of relevant prior history/indications, ${ }^{*}$ image viewing and manipulation, ${ }^{*}$ pattern recognition, ${ }^{*}$ interpretation, ${ }^{*}$ recommendation, clinical record keeping and reporting, ${ }^{*}$ consultation (on interpretations and 
management/workup strategy), *obtaining advice, and *receiving feedback.

Non-practice-oriented activities. Non-practice-oriented activities include ${ }^{*}$ personal and research database update, including personal teaching files; accounting and finance; *analysis and presentation of data; note taking and manuscript preparation; communication with colleagues; *continuing education; and *teaching and preparation of instructional materials.

As can be seen, this distinction is somewhat artificial, and there are a number of potential interactions between the classes of activity. Furthermore, most of the activities in both classes (those indicated by ${ }^{*}$ ) can benefit from augmentation by access to information resources for education and decision support.

\section{Educational and Decision-Support Applications}

We list in Table 1 some of the kinds of applications that may be useful for education and decision support. While not all of these are currently available in computer-based form, this category of applications is growing rapidly, and has potential for augmenting clinical practice. However, without consideration of consistent ways to access the multiple kinds of information resources they use, and an architecture into which these resources should fit, the proliferation of applications will be uncoordinated, redundant, and perhaps even daunting to potential users and developers.

Some patient care information system (PCIS) and radiology information system (RIS) implementations provide access to educational resources and decision aids themselves. PACS implementations may include image analysis and modeling capabilities. However, to the extent that these systems incorporate such capabilities, the educational and decision-support tools are typically duplicative of those offered through other means, having required reimplementation so as to allow access from within the clinical system. [For example, certain Harvard hospitals have implemented their own bibliographic search systems, drug information databases, and electronic mail systems. In part, this was for historic reasons. Nonetheless, because

Table 1.

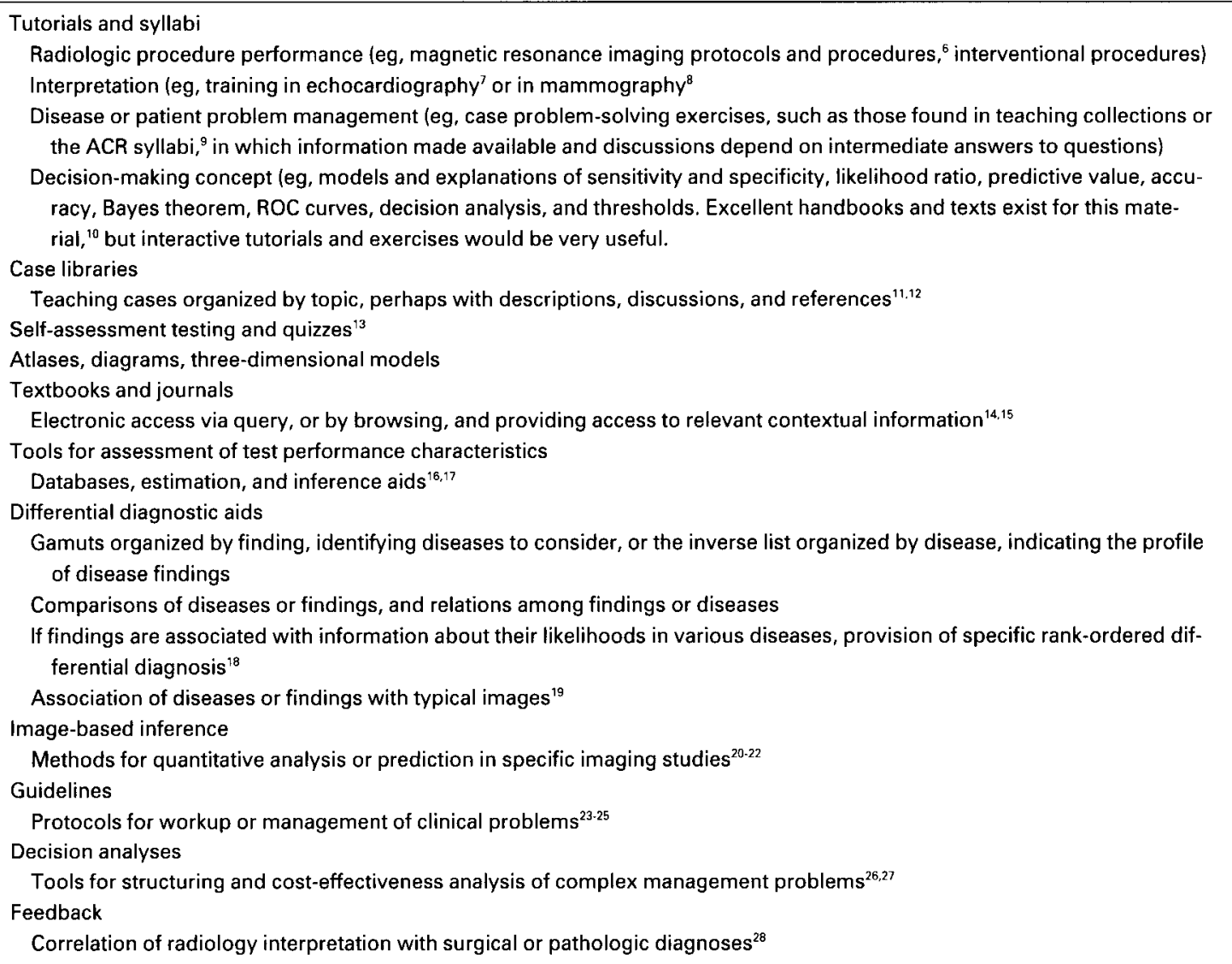


of the unique nature of the platforms for these systems, the institutions must continue to build (or reimplement) homemade versions of applications unless they address issues of participation in a network-aware "open" environment. This situation is certainly not unique to Harvard. The exception to this is PACS, to some extent, because these newer systems tend to have a more modular, network-aware design, and may use external servers for specialized functions.]

We emphasize the active proliferation of information resources external to any particular clinical system, and the potential value of an environment that can provide access to both clinical information systems functions as well as these capabilities, ideally in a well-integrated, consistent fashion.

\section{THE DESYGNER ARCHITECTURE}

\section{Previous Work}

The current DeSyGNER project has evolved from a series of experiments in building tools to integrate various educational resources and decision aids on a personal computer (the Macintosh, Apple Computer Corp, Cupertino, CA). In 1985 we developed Explorer- $1,^{29}$ a prototype information access environment and authoring tool, and CASPER (Computer-Aided Selection of Procedures and Evaluation of Results) ${ }^{30} \mathrm{a}$ knowledge base aimed at providing guidelines for imaging workup for common clinical problems. The content of CASPER was based on both prior compilation of clinical expertise in handbook form ${ }^{23}$ and previous work on clinical decision aids. ${ }^{17}$ CASPER presented clinical workup strategy for a set of common clinical problems, including a recommended clinical algorithm for each problem, and data pertaining to pertinent tests, in a multiwindow format. Other windows were used to provide bibliographic references, a dynamic "what if" analysis for estimating potential impact of a test for a specific patient, simulations to illustrate probabilistic decision-making concepts involved in test assessment, ${ }^{31}$ and an overview map used as an aid to navigation and browsing through the knowledge base. Content could be accessed by either hyperlinking between windows (display of windows containing additional information content by selection of a "hot spot" or active area in a current window), by restricted natural language query, or via the overview map. An evaluation study demonstrated the ease of use of CASPER for answering queries in its domain. (However, CASPER was of limited practical usefulness when operating as a stand-alone application, and the handbook on which it was based is now out of date. Updating it and providing its capabilities in a more integrated fashion in our clinical environment are now being explored as a possible component of a physcian order-entry application being developed at our institution for the patient care information system.)

A primary motivation was to explore the requirements for an information environment that would facilitate access to multiple databases and knowledge bases, encompassing a variety of information resource types, for purposes such as those described earlier. Through CASPER, Explorer-1 incorporated text, graphics, simulations, "what if" analyses, bibliographic lists, and an overview window. Explorer-1 was succeeded by an object-oriented reimplementation known as Explorer-2, which provides a software framework for access to information resources in a more extensible fashion. ${ }^{32}$ Under Explorer-2 we incorporated access to a differential diagnosis knowledge base (QMR, ${ }^{18}$ through the generous cooperation of R. Miller and his colleagues at the University of Pittsburgh) and to content from electronic textbooks (Scientific American Medicine, and Williams and Wilkins' Principles of Ambulatory Medicine, through the cooperation of the respective publishers). We also included a tool for high-resolution image manipulation and viewing, ${ }^{33}$ and a tool for dynamic adjustment of the presentation of clinical algorithms, ${ }^{25}$ to facilitate their adaptation to more complex clinical problems and interface with clinical information systems.

Explorer-2 supported several application paradigms or methods of use of the information resources, among them access to resources via query, hypertext linkage, and overview browsing. It also included two other ways to compose information into applications: a "page layout" method for constructing spatial arrangements of information resources, and a "book layout" method for establishing hierarchical organizations of resources to simulate an electronic book. 


\section{DeSyGNER}

Current work in DeSyGNER attempts to generalize our experience from Explorer-1 and Explorer-2 to provide higher level application construction capabilities for nonprogrammer authors. The main purpose is to make it easier to build applications by providing composition methods or organizational paradigms for constructing certain classes of applications.

DeSyGNER applications are composed from a set of modular information resources, termed entities. For each of the application paradigms, specific visual and organizational metaphors, or composition methods, are supported. Authoring and user interfaces for these composition methods are termed shells. Using these shells, and the composition methods they implement, the author may incorporate appropriate entities and specify the means by which these resources communicate with each other. DeSyGNER implements the above concepts by providing a set of kernel services that allow entity types to be defined, implemented, and shared. This is described in more detail elsewhere. ${ }^{2,3}$

Modular building blocks: Entities. The definition of entity is somewhat arbitrary in that it reflects the level of granularity at which a developer wishes to partition the information tasks of a potential application. The underlying premise is that such partitionings, for a number of applications, tend to result in entities of somewhat similar nature, and evolve to become classes of entities with well-defined behaviors. An entity tends to be an information resource with a self-contained set of actions that it performs, which do not depend on other entities. It may have linkages to or participate with other entities in an application, but the entity accomplishes a particular task on its own.

Entities are constructed using the methodology of Object Oriented Programming (OOP) ${ }^{34}$ They have in common a suite of capabilities that allow them to intercommunicate with other units. Entities are responsible for functions such as retrieval, display, and update (eg, in response to user interaction events pertaining to them, response to links coming into them, provision of links exiting from them, and support for associated overlays and index terms). These functions are termed methods of the entity object. Because of the inheritance made possible by OOP, entities form an open-ended class hierarchy.

Figure 2 depicts examples of various kinds of information resources that have been implemented that may be useful in education and decision-support applications. As may be seen, not only the content domains of such resources,

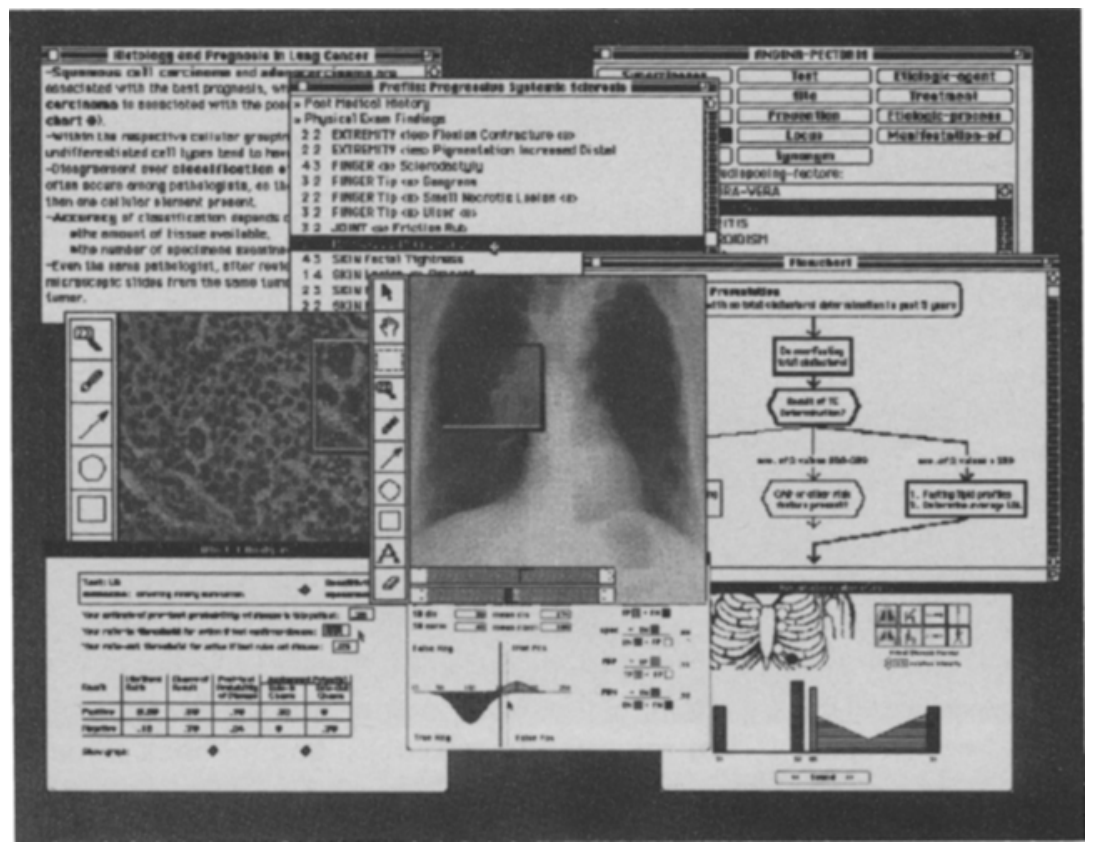

Fig 2. Examples of entities that have been implemented for various applications we have developed, illustrating their possible range. Shown here from left to right are a hypertext display, a disease profile list, a conceptualtaxonomy browser, a color pathology slide (reproduced here in black and white) a "200m image" (with gray scale manipulation and magnification capabilityl, a dynamic flow chart display, a "what if" spreadsheet, a graphical simulation of test distributions and performance characteristics, and a graphical display of auscultation findings. 
but also their forms, functions, and purposes, can be quite varied.

Application composition methods. Applications are constructed by interrelating various entities, according to a particular paradigm that determines how they are organized and by the protocols through which control is transferred from one information resource to another. For example, electronic book browsing can be supported by a paradigm in which entities are organized into a hierarchical framework. Page layout is a paradigm that allows entities to be organized spatially. Hyperlinking allows selection by a user of designated regions of an information resource to activate other information resources. A tutorial can be constructed by including the ability to perform conditional evaluations with hyperlinking. An algorithm visual metaphor can be used to organize information resources (eg, for portrayal of guidelines with explanations, or for visualizing the structure of a tutorial). Query can be supported by organizing information resources according to index terms, which may be selected by the user.

Composition methods create complex entities out of simpler entities. Hierarchical book layout, spatial page layout, and algorithmic flow control compositions are examples of such complex entities. These may be incorporated recursively as participants in other compositions.

These various paradigms can be combined; for example, the entities contained in a hierarchic book layout or a page layout may perform hyperlinking, and they may be retrieved by query.

Figure 3 schematically depicts a variety of possible composition methods or paradigms for applications. ${ }^{35}$ Those currently implemented include hyperlinking, spatial, hierarchic, queryoriented, and protocol-based paradigms. Figure 4 is an example of a radiology teaching file case constructed with DeSyGNER that uses zoom images, color pictures, text, and button elemental entities, and query, page layout, hyperlinking, and book layout composition methods.

Linking. The link is a particular form of composition in which an action or event (userinitiated or system-initiated) that occurs in the context of one entity generates a message causing directional or temporal progression to another entity's context. A hyperlink is an example of this. The generalization of a link is a script, although we have thus far avoided providing scripting by incorporating capabilities for defining necessary components of a link through direct manipulation authoring.

This is largely done via "dependents," features of the target entity that are incorporated in a link. Dependents convey specific view or other state information that governs the way the triggered entity will appear or the processing that it will perform. For example, an image is displayed by an invoked image entity with a specific cropping, magnification, brightness and contrast, and overlays associated with a link. From another link, this same image might have a very different appearance.

Extensibility, adaptation, and evolution of standards. Both individual entity types and methods of composition can be extended and adapted to changing requirements. If a new entity type or composition method is needed, it is separately implemented. The class hierarchy of entities provides methods for its various capabilities, which must be specifically overridden or modified to reflect the new behavior.

Through evolution of entities and their communication needs, we develop standardized protocols for communication between them. The OOP approach allows each object to implement its own internal data storage and access methods, and only requires that objects communicate with each other in agreed upon ways. We do not impose requirements on the internal structure of an entity nor require that it conform to standards for databases, image formats, and so forth. If a new image format is needed, the entity to manipulate this becomes a specialization of an existing image entity. Thus, we are able to begin without standards, unless they already exist. However, in the process of evolution we can expect convergence on standards.

\section{FUTURE GOALS AND DIRECTIONS}

We intend DeSyGNER to be a prototype of a future information technology architecture in which information modules (entities) are provided by researchers, developers, domain experts, and information suppliers, the producers, and in which applications users and developers are the consumers of these entities. The emphasis of the information environment is to couple 
the producers and the consumers through composition and linkage. This concept is intrinsically a group cooperative enterprise. We believe that the implementation of systems to support the needs of the health-care professional should be viewed in this light.

A primary long range goal is a single environment in which both clinical and nonclinical applications can be supported in a consistent fashion. The development of a clinical interface is thus an important priority, and is the current focus of a number of our projects. Applications we are pursuing include (1) designation during image interpretation of images to be captured for teaching and research, (2) access to differential diagnosis for findings as well as associated archived images, (3) multiperson consultation about clinical cases supporting image viewing and annotation, (4) use of clinical algorithm displays to guide data entry for radiology reporting, and (5) use of algorithms to guide entry of physician orders for radiologic procedures.

Clinical applications are currently implemented on a variety of platforms, typically not $\mathrm{PC} /$ workstation-based, and provide often incompatible environments. This problem is ameliorated somewhat, in that clinical systems can usually be accessed by terminal emulation from the PC/workstation. Thus, simply by implementing a terminal emulation entity, DeSyGNER can be host to both clinical applications as well as multimedia education and decision support.

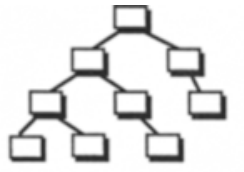

hierarchic, structured

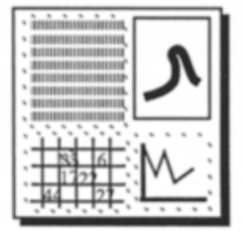

visual context-oriented
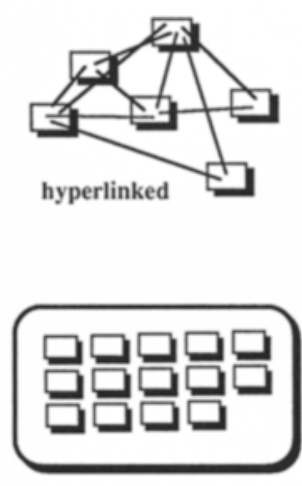

storyboard or slide sorter
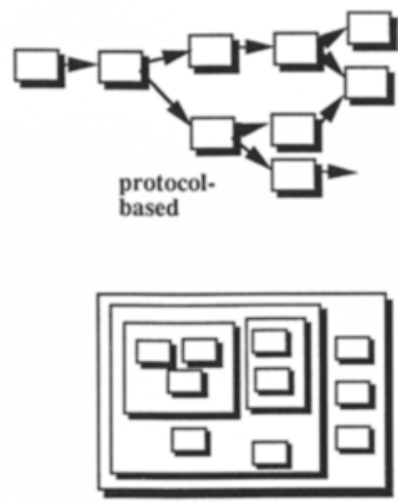

nested collection
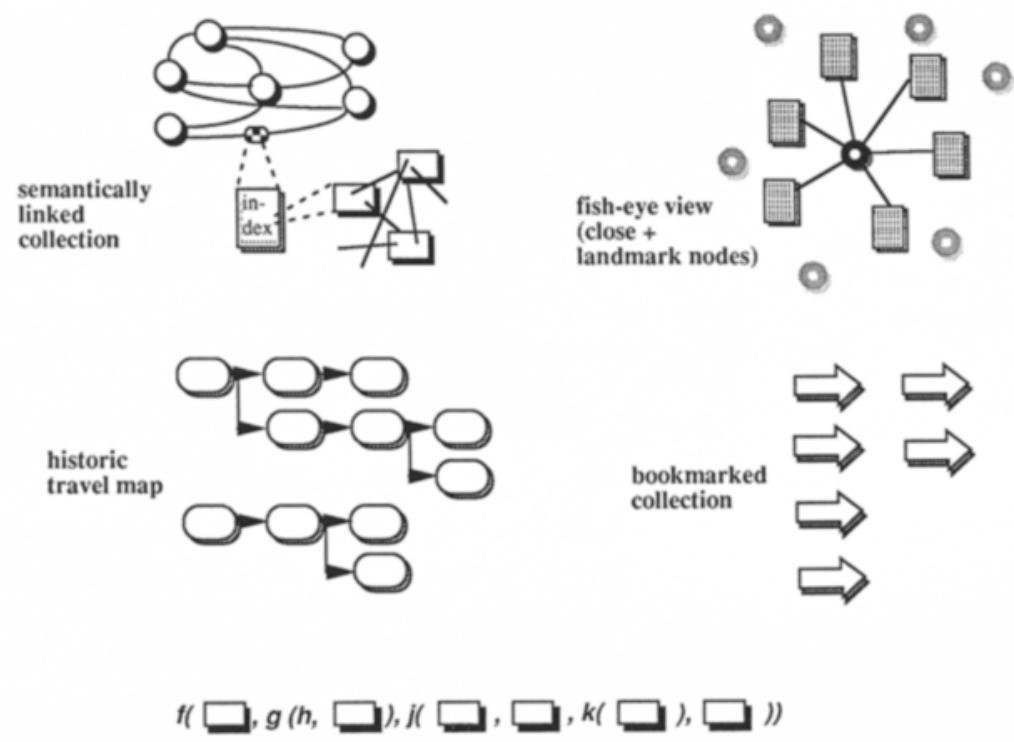

functional dependencies

Fig 3. Schematic diagrams showing principal composition methods or paradigms for constructing applications from modular entities. Individual boxes can be comprised of any entity type. (Adapted with permission. ${ }^{35}$ ) 
Fig 4. A radiology teaching file case constructed with $\mathrm{De}$ SyGNER, which uses zoom images, color pictures, text, and button elemental entities. The upper left window is retrieved by means of a query on the type of case, and represents an example of a page layout composition method. Hyperlinks from buttons or highlighted words are used to bring up other windows leg, that on the lower left and on the right). The discussion window to the right illustrates a section of an electronic book, constructed with the book layout composition method. (Digitized pathology images are courtesy of Daniel Alonso, MD, and Steven Erde, MD, Cornell University Medical Center, New York. NY. These images, normally in color, are reproduced in black and white.)

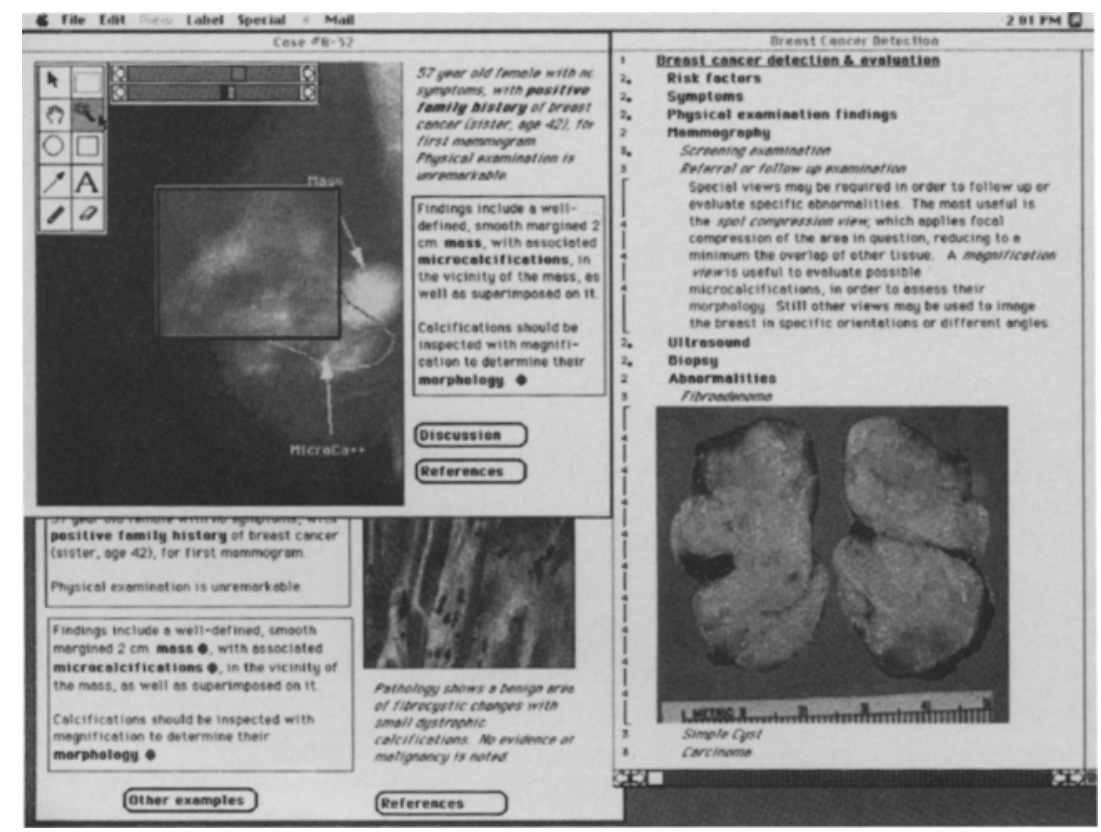

However, actual functional integration of the clinical application and the education and decision-support resources does not occur.

What is needed to achieve actual functional integration? The disparity between architectures of many existing, operational clinical systems, which have evolved over a long history, and newer workstation-based, network-aware software engineering methodologies points up the importance of developing an evolution strategy. We cannot simply scrap and replace functioning systems. We suggest that the strategy required is one in which clinical systems progress toward assuming primarily back-end transaction processing roles, furnishing data to workstation-based front-end systems with which users interact.

We describe here certain methodologies and technologies that we believe to be important, which are being incorporated in our current work on the DeSyGNER architecture.

\section{Object-Oriented Design}

Object-oriented design (OOD) is a methodology for describing the function of a system by a process of characterizing the individual objects in the system, concrete as well as abstract, computer-based as well as human, in terms of their specific roles and responsibilities, as well as by the other objects with which they collabo- rate or communicate.$^{36}$ Emphasis is on delineation of the classes of such objects, which share common properties, forming inheritance trees, on the functions that each object must perform, and on the communication protocols specific to those tasks.

Rationale for this methodology is its emphasis on defining channels of communication. If information tasks could be invoked by welldefined messages and produced well-defined effects, then any task could be invoked from any context with anticipated results. OOD essentially provides an external functional specification for an information task; the information task may be implemented in whatever way desired as long as its external behavior and communication can be made known.

We believe that OOD is a useful methodology for describing the work of a professional such as the radiologist, because it is unbiased by existing system implementations, and concentrates on the channels of communication that must be realized. This process can serve to identify specific information objects needed and the information that must be passed between them. Our belief is that as we extend the DeSyGNER environment to include clinical functions, the OOD process will help us define the specific communication requirements be- 
tween clinical, educational, and decision-support activities.

\section{Open Systems}

To the extent possible, one desires that information resources be accessible on a variety of workstation platforms. Further it is important that the information resources themselves respond appropriately to requests from these various platforms, independent of the kinds of systems on which they are implemented. The above can be achieved by (1) adhering to client-server communication for a set of transactions that must traverse dissimilar systems and (2) separating the rendering of user interface (eg, the Graphical User Interface [GUI]) from the processing and view architecture of the content. Development environments and tools for accomplishing these goals are beginning to appear. ${ }^{37}$

\section{Broadband Communication}

The modular units from which applications are composed are seen as potentially being distributed across a network, accessible by any workstation, rather than requiring an external host information system in order to gain access to them. The workstation is responsible for flow control, not a host information system. This would enable PCIS, PACS, RIS, PC/workstation-based, and remote modules to all be incorporated into applications as needed. Broadband networks are expected to approach gigabitper-second speeds over the next several years, stimulated by private initiatives of the telecommunications industry, ${ }^{38}$ and by the High Speed Computing Initiative. ${ }^{39,40}$

\section{Client-Server Relationships}

The primary means by which disparate clinical systems will be able to operate in conjunction with a $\mathrm{PC} /$ workstation front-end is by implementation of client-server relationships between the two. Initial access to clinical information in DeSyGNER can be done by an entity supporting simple terminal emulation to display the necessary data. As needs for data passing become further defined, the RIS or other information system should gradually be modified so that its various user applications provide modes of access that separate front-end from backend, allowing a user interface to be constructed on a workstation, for example, to replace a terminal-based user interface for a patient scheduler. As required by applications such as patient scheduling, examination inquiry, and reporting, the RIS would need to be able to respond to several classes of transaction requests (eg, for retrieving specific data, for data validation and checking, and for storing data). Gradually, the RIS would become a transaction processing server for workstation clients. For example, a radiology information system should be able to provide a basic set of data on a transaction basis, without regard to formatting, such as a list of examinations for a patient, a report of a particular examination, a schedule, or the locations of the image folders. A finite number of such transactions need to be provided by an information system.

\section{Communication Protocols}

Another task that must be performed, not only for client-server communication but for peer-peer entity communication, is a specification of message formats for passing data. Clearly, this is needed for the transaction processing involved in building an interface to an RIS or PACS server, for example. Generally, this will be at a finer granularity than $\mathrm{HL} 7^{41}$ or ACRNEMA ${ }^{42}$ because we refer to the communication for individual transactions, not entire systems.

The specification of message content of links becomes particularly important as entities must communicate specific data to each other, and where the specific presentation or processing of an entity depends not only on the occurrence of a link to it, but on information pertaining to context or state of the application, including processing carried out by previous entities. As noted previously, current links in DeSyGNER must know little about the source entities, only about the presentation of the target. Yet many examples exist in which more information must be conveyed. A differential diagnosis expert system, for example, should expect to receive clinical findings from a patient inquiry module. A "what if" analysis entity for a test must know which test is of interest. The results of analysis 
should be able to be passed back to a clinical report entity.

Links should be able to be specified as intentional or extensional ${ }^{43}$ in terms of whether explicit targets are designated versus criteria for selecting them. We need to further enhance the definition of links to provide processing of an associated procedure, including evaluation of conditionals, and to carry parameters in its message. We may need to involve full scripting capability, but we hope to largely avoid this by allowing the semantic associations keyed to entities to provide access to a set of methods for constructing messages and passing data that are appropriate to the author's intention.

\section{Vendor Participation}

An important ingredient for the integrated information environment of the future is recognition by the developers of clinical information systems applications that they must evolve to support such an environment. In lieu of complete reimplementation, this will be done primarily, as discussed earlier, by evolving separation of the various transactions they provide into front-end and back-end components.

Ideally, through the above approach, and by concentration on the message protocols, we can separate the way in which an application appears to a user and the way in which data are provided to or sent from the application. This issue is important, whether we consider viewing an image, retrieving data from a database, or reading an electronic book. In its host environment, the image may be in a variety of formats (eg, TIFF, PICT, ACR-NEMA), but should be capable of being sent in a generic way, on recognition of a request for it. Clinical data may be stored in a variety of schemas, but should be sent in a standard way as a result of a transaction interface message. The requirement for SQL and other syntaxes for retrieval should be determined at the level of the specific database, but the message must be at a more generic level. An electronic book may be stored on a CDROM in a custom format. The user interface for accessing it should be able to request a hierarchical section of it without regard to that internal format. Wiederhold et $\mathrm{l}^{44}$ have introduced the notion of mediators as intermediate layers in an information environment that translate an applications request for a particular view of data or knowledge into a set of database-specific operations for retrieving and organizing it.

\section{ACKNOWLEDGMENT}

The contributions to the work described here by other present and former members of the Decision Systems Group are gratefully acknowledged, particularly those of Thomas Abendroth, MD, Bryan Bergeron, MD, Lawrence Cope, Stephan Deibel, William Hersh, MD, Andre Marquis, James McClay, MD, Robert McClure, MD, Edward Pattison-Gordon, Jan Snydr-Michal, Lincoln Stein, MD, and David Tarabar.

\section{REFERENCES}

1. Greenes RA: "Desktop knowledge": A new focus for medical education and decision support. Methods Inf Med 28:332-339, 1989

2. Greenes RA, Deibel SRA: The DeSyGNER knowledge management architecture: A building block approach based on an extensible kernel. Artif Intell Med 3:95-111, 1991

3. Deibel SRA, Greenes RA, Snydr-Michal JT: DeSyGNER: A building block architecture fostering independent cooperative development of multimedia knowledge management applications, in: Proceedings of the Fourteenth Annual Symposium on Computer Applications in Medical Care (SCAMC), Washington, DC, November 1990. New York, NY, IEEE Computer Society Press, 1990, pp 445-449

4. Greenes RA: The radiologist as clinical activist: A time to focus outward, in Mun SK, Greberman M, Hendee WR, et al (eds): Proceedings of the First International Conference on Image Management and Communication in Patient Care: Implementation and Impact (IMAC 89),
Washington DC, June 1989. Los Alamitos, CA, IEEE Computer Society Press, 1989, pp 136-140

5. Greenes RA, Shortliffe EH: Medical informatics: An emerging academic discipline and institutional priority. JAMA 263:1114-1120, 1990

6. Posteraro RH, Blinder RA, Herfkens RJ: MR Tutor: A program for teaching the interdependence of factors which influence signal intensity in magnetic resonance imaging. Comput Med Imag Graph 13:393-406, 1989

7. Jaffe CC, Lynch PJ, Smeulders AWM: Hypermedia techniques for diagnostic imaging instruction: Videodisk echocardiography interpretation. Radiology 171:475-480, 1989

8. Getty DJ, Pickett RM, D'Orsi CJ, et al: Enhanced interpretation of diagnostic images. Invest Radiol 23:240252,1988

9. Siegel BA: Professional Self Evaluation Continuing Education Program (continuing series). Reston, VA, American College of Radiology 
10. Sox H, Blatt MA, Higgins MC, et al: Medical Decision Making. Boston, MA, Butterworths (1988).

11. Arenson RL, Greenes RA, Allman R, et al: Digital radiology image learning library. Proceedings of the 75 th Annual Meeting of the RSNA, Chicago, IL, November 20, 1989 (abstr)

12. The ACR Videodisc Library (continuing series). Reston, VA, American College of Radiology

13. Bergeron BP, Morse AN, Greenes RA: A generic neural network-based tutorial supervisor for computer aided instruction, in: Proceedings Fourteenth Annual Symposium on Computer Applications in Medical Care (SCAMC), Washington, DC, November 1990. New York, NY, IEEE Computer Society Press, 1990, pp 435-439

14. Hersh WR, Greenes RA: SAPHIRE-An information retrieval system featuring concept matching, automatic indexing, probabilistic retrieval, and hierarchical relationships. Comput Biomed Res 23:410-425, 1990

15. Knowledge Finder, Aries Systems Corporation, North Andover, MA. [Note: This is one example of a large and growing number of commercial and noncommercial products that perform MEDLINE and other bibliographic searches, on bibliographic databases and on full text documents, via network or CDROM databases.]

16. Greenes RA: Interactive microcomputer-based graphical tools for physician decision support: Aids to test selection and interpretation and use of Bayes theorem. Med Decis Making 3:15-21, 1983

17. Greenes RA: Computer-aided diagnostic strategy selection. Radiol Clin North Am 24:105-120, 1986

18. Miller RA, McNeil MA, Challinor SM, Masarie FE, Myers JD: The Internist-1/Quick Medical Reference project-Status Report. West J Med 145:816-822, 1986

19. Swett HA, Fisher PR, Cohn AI, Miller PL, Mutalik PG: Expert system-controlled image display. Radiology 172:487-493, 1989

20. Sandor T, Kalender WA, Hanlon WB, et al: Spinal bone mineral determination using automated contour detection and dual energy CT. Proc Society of Photo-optical Instrumentation Engineers 555:188-194, 1985

21. McNeil BJ: Ventilation-perfusion studies and the diagnosis of pulmonary embolism: concise communication J Nucl Med 21:319-323, 1980

22. Greenes RA: OBUS: A measurement, calculation, reporting, filing and retrieval computer system for obstetrical ultrasound examinations. Radiology 144:879-883, 1982

23. McNeil BJ, Abrams HL (eds): The Brigham and Women's Hospital Guide to Diagnostic Imaging. Boston, MA, Little, Brown, 1986

24. Margolis CZ: Uses of clinical algorithms. JAMA 249:627-632, 1983

25. Abendroth TW, Greenes RA, Joyce EA: Investigations in the use of clinical algorithms to organize medical knowledge, in: Proceedings of the Twelfth Annual Symposium on Computer Applications in Medical Care (SCAMC), 1988. New York, NY, IEEE Computer Society Press, 1988 pp 90-93 [Subsequent modifications to this tool have been made by $\mathrm{J}$ McClay, unpublished.]

26. Pauker SG, Kassirer JP: The threshold approach to clinical decision making. N Engl J Med 302:1109-1117, 1980
27. Lau J, Kassirer IP, Pauker SG: Decision Maker 3.0. Improved decision analysis by personal computer. Med Decis Making 3:39-43, 1983

28. Greenes RA, Bauman RA, Robboy SJ, et al: Immediate pathologic confirmation of radiologic interpretation by computer feedback. Radiology 127:381-383, 1978

29. Greenes RA: Knowledge management as an aid to medical decision making and education: The Explorer-1 system, in: Proceedings of MEDINFO 86, Washington DC, October 1986. Amsterdam, The Netherlands, Elsevier, 1986, pp $95-899$

30. Greenes RA, Tarabar DB, Krauss M, et al: Knowledge management as a decision support method: A diagnostic workup strategy application. Comput Biomed Res 22:113135,1989

31. Pollack MA, Greenes RA: A pictorial simulation construction kit for enhancing knowledge-based learning, in: Proceedings of MEDINFO 86, Washington DC, October 1986. Amsterdam, The Netherlands, Elsevier, 1976, pp $887-890$

32. Greenes RA, Tarabar DB, Slosser ET: Explorer-2: A multi-modal knowledge management framework for medical education and decision support, in: Proceedings of the Thirteenth Annual Symposium on Computer Applications in Medical Care (SCAMC), Washington, DC, November 1989. New York, NY, IEEE Computer Society Press, 1989, pp 1024-1025

33. Stein LD, Snydr-Michal JT, Greenes RA: Realistic viewing and manipulation of radiographic images on a personal computer-a digital interface for educational and other applications. J Digital Imaging 4:169-176, 1991

34. Cox B: Object-Oriented Programming: An Evolutionary Approach. Reading, MA: Addison-Wesley, 1986

35. Greenes RA: Approaches to sharing and collaboration through modular systems design: A focus on knowledge management (Keynote paper), in: Timmers T, Blum BI (eds): Software Engineering in Medicine: Proc IMIA Working Conf. Amsterdam, The Netherlands, Elsevier Science, 1991 (in press)

36. Booch G: Object Oriented Design with Applications. Redwood City: Benjamin-Cummings, 1991

37. Rochkind MJ: XVT: A virtual toolkit for portability between window systems. Proceedings of the Winter 1989 USENIX Conference, pp 151-163

38. McGarty TP: Mutimedia communications technology in diagnostic imaging. Invest Radiol 26:377-381, 1991

39. Kleinrock L: Technology issues in the design of the NREN. Information Infrastructures for the 1990's, Workshop/Symposium VIII, John F. Kennedy School of Government, Harvard University, November 29-December 1, 1990

40. Committee on Physical, Mathematical, and Engineering Sciences: Grand Challenges: High Performance Computing and Communications. The FY 1992 U.S. Research and Development Program. Office of Science and Technology Policy, US Government Report, Washington, DC, 1991

41. Health Level Seven Standards: An Application Protocol for Electronic Data Exchange in Health Care Environments, Version 2, HL7. Philadelphia, PA, 1988, pp 1-15

42. ACR-NEMA Digital Imaging and Communications Standard. Publication No. 300-1985, National Electrical 
Manufacturers Association, 2101 L St, NW, Suite 300, Washington, DC 20037

43. DeRose SJ: Expanding the notion of links, in Akscyn R (ed): Proceedings of Hypertext 1989, Philadelphia, PA, November 1989. New York, NY, ACM, Special IssueSIGCHI Bulletin, 1989, pp 249-258
44. Wiederhold G: Future architectures for information processing systems, in Rishe N, Navathe S, Tal D (eds): Proceedings of Parbase-90, International Conference on Databases, Parallel Architectures, and their Implementation. IEEE Computer Society Press, Washington DC, March 1990, pp 160-176 